\title{
Mapping multiple genetic loci associated with Ceratomyxa shasta resistance in Oncorhynchus mykiss
}

\author{
Krista M. Nichols ${ }^{1,3, *}$, Jerri Bartholomew ${ }^{2}$, Gary H. Thorgaard ${ }^{1}$ \\ ${ }^{1}$ School of Biological Sciences and Center for Reproductive Biology, Washington State University, Pullman, \\ Washington 99164-4236, USA \\ ${ }^{2}$ Center for Fish Disease Research, Department of Microbiology, Oregon State University, Corvallis, \\ Oregon 97331-3804, USA
}

${ }^{3}$ Present address: National Oceanic and Atmospheric Administration, Northwest Fisheries Science Center, Conservation Biology Division, 2725 Montlake Blvd E, Seattle, Washington 98112, USA

\begin{abstract}
Ceratomyxa shasta is enzootic to some rivers of the Pacific Northwest and California, and has caused extensive mortalities of salmonids, the only known host of the actinosporean stage of the pathogen. Mortalities from C. shasta are induced by severe inflammation and necrosis of the intestinal tract, and no therapeutants have been discovered for the treatment of ceratomyxosis. Inheritance studies by previous investigators suggested that $C$. shasta resistance is genetically controlled, but none have revealed the number and locations of gene regions associated with resistance. Here, we characterized differences in resistance among clonal lines, and used line crosses between clonal lines with resistance differences for a genetic analysis of $C$. shasta resistance in Oncorhynchus mykiss, following a short term in situ exposure to the pathogen. We found that Oregon State University $(\mathrm{OSU}) \times$ Arlee $(\mathrm{ARL})$ clonal hybrids were highly susceptible, while OSU $\times$ Clearwater $(\mathrm{CW})$ hybrids were highly resistant to $C$. shasta. Doubled haploids, produced by androgenesis from an $\mathrm{F}_{1}$ hybrid between the OSU and CW lines, were evaluated for the co-segregation of molecular markers and resistance phenotype. Amplified fragment length polymorphic (AFLP) markers, used to construct a genetic linkage map in this cross, were tested for associations with resistance expressed as absolute survival (binary trait) and days to death (survival analysis). Segregation of the resistance phenotype suggested that the resistance was inherited as a single Mendelian locus, but the binary trait failed to map to a single genomic location. Markers identified in single-marker analyses were used in multiple-regression model selection, within both the linear regression and Cox proportional-hazards survival analysis frameworks, to determine the number and potential location of loci simultaneously contributing to variation in the survival phenotypes of doubled-haploid progeny. We found multiple genomic loci associated with $C$. shasta resistance through these models, confirming studies by previous investigators that suggested $C$. shasta resistance is polygenic.
\end{abstract}

KEY WORDS: Ceratomyxa shasta $\cdot$ Oncorhynchus mykiss $\cdot$ Survival analysis · Quantitative trait loci Resale or republication not permitted without written consent of the publisher

\section{INTRODUCTION}

Ceratomyxa shasta is a parasite enzootic to some watersheds of the Pacific Northwest and California, and can be a significant cause of mortality among salmonids that migrate through, reside in, or are reared in waters in which the infectious stage of
C. shasta is present (Bartholomew 1998 and references therein). C. shasta is a myxozoan parasite that requires 2 hosts for complete development of its life cycle. Although pathogenesis in salmonids has been recognized since the 1940s (Bartholomew 1998 and references therein), the alternate host was only recently identified as the freshwater polychaete worm Man- 
ayunkia speciosa (Bartholomew et al. 1997). The actinosporean stage of $C$. shasta, developing in the polychaete worm, releases actinospores into the water column that subsequently infect the intestinal tracts of susceptible salmonid hosts. Severe inflammation and degradation of the intestinal tract as a result of C. shasta exposure, followed by mortality, is called ceratomyxosis. The pathogenesis of $C$. shasta has been well described, but no treatment for ceratomyxosis has been found. The only current method for control of ceratomyxosis is avoidance of rearing or stocking fish in water sources in which the infectious actinosporean stage is present (Bartholomew 1998, Whipple et al. 2002).

Both inter- and intra-specific variation in Ceratomyха shasta resistance has been demonstrated in natural and hatchery populations of salmonids (Bartholomew 1998 and references therein), and this variation is presumably a result of the selective forces of $C$. shasta in waters in which individuals are exposed during their life cycle. Many salmonid populations that migrate through or are reared in enzootic areas are more resistant than populations from outside these areas (Buchanan et al. 1983, Hemmingsen et al. 1986). A comprehensive suite of inheritance studies in Oncorhynchus mykiss has revealed that resistance is indeed genetically controlled (Ibarra et al. 1992, 1994, Bartholomew et al. 2001). Ibarra et al. $(1992,1994)$ concluded that resistance is conferred by multiple loci, on the basis of segregation patterns observed in $F_{1}, F_{2}$, and backcross families produced in a cross between resistant and susceptible strains. A simple Mendelian model of inheritance failed to explain the differences observed in both absolute mortality and days to death. With comprehensive molecular maps available for O. mykiss (Young et al. 1998, Sakamoto et al. 2000, Nichols et al. 2003), the tools are now available to estimate the number and location of gene regions associated with $C$. shasta resistance.

In this study, we characterized Ceratomyxa shasta susceptibility in clonal lines of rainbow trout and performed a systematic search of the genome, using line crosses, to detect the number and potential locations of loci associated with C. shasta resistance. Geographic origins of the outbred populations used to establish our Oncorhynchus mykiss clonal lines suggested that some may be more resistant than others to C. shasta. Based upon the characterization of differences in resistance among our clonal lines, doubled-haploid progeny produced from a hybrid between resistant and susceptible lines were used for a genetic analysis of $C$. shasta resistance. This type of study is similar to quantitative trait loci (QTL) analysis, whereby a genetic linkage map is used to systematically search for genomic positions with significant statistical associations with quantita- tive characters (Lander \& Botstein 1989). Here, we tested for the co-segregation of molecular markers with C. shasta resistance or susceptibility expressed as absolute survival (binary trait) and as days to death (survival analysis).

\section{MATERIALS AND METHODS}

Line crosses. To characterize resistance of isogenic lines to Ceratomyxa shasta, clonal hybrids were produced by fertilizing eggs from an Oregon State University (OSU) clonal individual with sperm from each of the Clearwater River (CW) and Arlee (ARL) clonal lines, in January 1999. The OSU clonal line originated from a research hatchery at Oregon State University and is a Shasta-type rainbow trout. The CW line originated from a hatchery stock of steelhead trout from the Dworshak National Fish Hatchery (Ashakha, Idaho) on the Clearwater River, and the ARL line from Arlee National Fish Hatchery (Arlee, Montana). Doubledhaploid progeny were produced by androgenesis (Parsons \& Thorgaard 1984, Young et al. 1998) from a cross between the OSU and CW lines for the genetic analysis of C. shasta resistance in February 2000. Briefly, eggs from 3 outbred female rainbow trout obtained from Troutlodge (Sumner, Washington) were irradiated to destroy the maternal nuclear genetic material. Eggs were fertilized with sperm from an $F_{1}$ hybrid male produced from a cross between the OSU and CW clonal lines. A heat shock was performed prior to the first embryonic cleavage to restore diploidy, resulting in doubled haploids with all-paternal inheritance. Clonal hybrids and doubled haploids were produced at Washington State University and reared in recirculating stack egg incubators until swim-up. Hybrids and doubled haploids were then maintained in 10 gallon recirculating tanks until they were shipped as fry to the Center for Fish Disease Research, Oregon State University, in January 1999 and July 2000, respectively.

Ceratomyxa shasta exposure and rearing conditions. C. shasta exposures were accomplished in situ in the Willamette River, Oregon in late summer, when the infectious stage of the organism is in greatest abundance (Bartholomew et al. 1997). Both clonal hybrids and doubled haploids were exposed in live cages. Clonal hybrids were exposed from 23 to 27 August 1999 and doubled haploids were exposed from 6 to 9 September 2000. At the same time, an outbred group of individuals from a known susceptible strain of fish was exposed as positive controls for C. shasta presence and susceptibility, since dose-exposure to C. shasta is difficult to quantify in situ, and laboratory exposure has not been possible (Bartholomew et al. 1997). At the 
end of the exposure, fish were transported from the river back to the Center for Fish Disease Research, where they were maintained in flow-through 251 tanks at $12^{\circ} \mathrm{C}$. Doubled-haploid progeny produced from different outbred females were maintained in separate cages and tanks to later assess any differences among the families in C. shasta susceptibility.

Evaluation of Ceratomyxa shasta resistance/susceptibility. Clonal hybrids and doubled haploids were monitored daily for mortalities following the exposure. Dead fish were removed and the day of death recorded. Presence or absence of $C$. shasta spores was evaluated by examination of posterior intestinal scrapings at $250 \times$ under a light microscope (Bartholomew et al. 1997). Post-exposure mortalities were monitored for $90 \mathrm{~d}$ for isogenic hybrids, and $103 \mathrm{~d}$ for doubledhaploid progeny. At the termination of the experiment, all surviving fish were euthanized with an overdose (500 $\mathrm{mg} \mathrm{l}^{-1}$ ) of tricaine methanesulfonate (MS-222; Argent Laboratories) and evaluated for the presence of C. shasta spores.

Genotyping and linkage mapping for doubled-haploid progeny. At the time of death, fin clips were taken from each doubled-haploid individual and stored in 95\% ethanol. DNA was extracted from fin clips using the Puregene DNA Isolation Kit (Gentra Systems). Doubled haploids were genotyped with amplified fragment length polymorphic (AFLP) markers as described by Robison et al. (2001). Twenty-seven EcoRI/MseI primer sets were used for genotyping. Markers for which less than $80 \%$ of individuals were scored were removed from the analysis. All markers were tested for significant departures from expected Mendelian segregation with a chi-squared test. Any markers significantly deviating from the expected 1:1 segregation were checked for genotyping errors and reliability, and were removed from the analysis if markers appeared unreliable due to difficulties in scoring. A linkage map was constructed using Mapmaker for Mac (v. 2.0, Dr. Scott Tingey, Dupont Agricultural Experiment Station), and Mapmaker/EXP (Lander et al. 1987), as described by Nichols et al. (2003). Markers were grouped together and ordered into linkage groups at a minimum log of the odds (LOD) score of 3.0 and maximum recombination of 0.4. Final linkage groups were drawn with Mapchart 2.1 (Voorrips 2002).

Statistical analyses. All statistical analyses were performed using the SAS System statistical software for Windows (SAS Statistical Institute), except where noted otherwise.

Clonal hybrids: Resistance or susceptibility differences between clonal hybrid groups were tested in 2 ways. Logistic regression (PROC LOGISTIC) was performed to test for differences in the proportion of individuals surviving between the $\mathrm{OSU} \times \mathrm{CW}$ and $\mathrm{OSU} \times$
ARL clonal hybrid groups. Survival analysis was also performed with the Cox proportional hazards model (PROC PHREG) to test for significant differences in the survival functions between OSU $\times$ ARL and OSU $\times$ CW. Type I error rate was set at $\alpha=0.05$ for comparisons between clonal hybrid groups.

Doubled haploids - summary statistics: Prior to tests for marker-trait associations, among-female family differences in 'proportion surviving' and 'days to death' were tested to determine whether maternal cytoplasmic factors may have significantly influenced overall differences in Ceratomyxa shasta resistance. Fish that died but did not have $C$. shasta spores in the intestinal tract were removed from all statistical analyses, as death could not be attributed to C. shasta infection. Logistic regression (PROC LOGISTIC) was performed to determine whether the proportion surviving the C. shasta challenge was significantly different among female families. Survival analysis was also performed using the Cox proportional-hazards model (PROC PHREG) to determine whether survivor curves for days to death were significantly different among females. Type I error rate was set at $\alpha=0.05$ for comparisons among doubled-haploid families.

Doubled haploids - tests for marker-trait associations: Single marker-trait associations were tested based on a decision tree of 2 hypotheses: (1) if equal proportions died and survived, the trait segregates as a single Mendelian locus and should map by traditional linkage mapping methods to the AFLP marker map; (2) given unequal proportions or the failure of the binary trait to map to a single locus, multiple genome regions may be involved. Multiple loci can be identified by evaluating associations of each marker singly with resistance expressed as a binary trait (died or survived), or in days to death, and subsequently evaluating significant and notable markers in multiple regression models to determine the number of loci significantly contributing to variation in resistance. To test the first hypothesis, a chi-square test was performed to test for differences in proportions of individuals that died and survived. Given equal proportions, the resistance/susceptibility binary trait was coded as a binary 'marker' and an attempt was made to map this marker to the AFLP marker linkage map. To do this, the AFLP framework marker map was used in an attempt to 'assign' the resistance/susceptibility marker to the map at LOD greater than 3.0 in Mapmaker/EXP (Lander et al. 1987). Given unequal proportions, or the failure of the binary trait to map with traditional single locus linkage mapping, several statistical models were used to test for the association of each single marker, with Ceratomyxa shasta survival data expressed as a binary term for died/survived or as days to death. In all models, the paternal or CW genotype was coded as 1 , 
and the maternal or OSU genotype was coded as 0 . For the binary survival phenotype, those that died were coded as 0 and those that survived were coded as 1 . Both linear (PROC REG) and logistic (PROC LOGISTIC) regression models were used to test for associations between each AFLP marker and the binary trait for survival or death. Linear regression has been shown to be robust from departures from normality, with approximately the same power as logistic regression models (Visscher et al. 1996, Rebai 1997) for identification of binary trait loci. Furthermore, linear regression has exhibited a better estimation of the recombination fraction (or distance) between single markers and the binary trait locus with close linkage (McIntyre et al. 2001). The Cox proportional-hazards model (PROC PHREG) was used to test for differences between survival curves (days to death) for AFLP marker genotype classes for each marker. To determine empirical $\mathrm{p}$-values for single markers, and the genome-wide threshold for significance with linear regression, permutation tests were performed with 10000 replicates using LRMapQTL in QTL Cartographer (Basten et al. 2002), and a framework of markers from the linkage map, removing all but one marker from clusters (markers with 0 centiMorgan [cM] distances). All single marker tests for significance were made with a Type I error rate of 0.05 , but notable associations are indicated for a Type I error rate less than or equal to 0.10 . Interval mapping by regression (Haley \& Knott 1992) was implemented in r/QTL (Broman et al. 2003) to ascertain the position of binary trait loci. Intermarker correlations, for those markers significantly or notably associated with survival, were calculated to

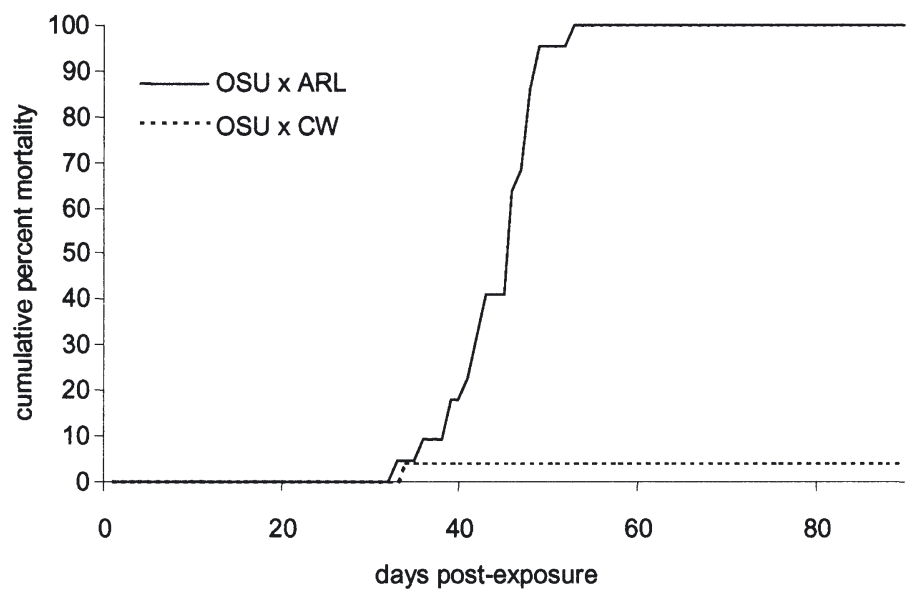

Fig. 1. Oncorhynchus mykiss infected by Ceratomyxa shasta. Cumulative percentage mortality for 2 clonal hybrid families. OSU $\times$ ARL $(-)$ is a cross between the Oregon State University (OSU) and Arlee (ARL) rainbow trout lines. OSU $\times \mathrm{CW}$ (---) is a cross between the OSU and Clearwater River (CW) rainbow trout clonal lines determine the propensity of markers on different linkage groups to segregate together in this study due to small sample size (or small number of segregation events).

Single-marker analysis results were used as a guide for marker inclusion into model selection procedures used to estimate the number of genomic loci associated with Ceratomyxa shasta resistance, expressed in both binary and days-to-death terms. In the case where significant and notable markers were clustered (showing the same genotype for each individual or $0 \mathrm{cM}$ distance), one marker was chosen to represent the cluster in multiple-marker model selection. Forward stepwise, backwards elimination (with p-values for including or removing markers both set at 0.15 ) model selection was used for both linear regression and survival models (PROC REG and PROC PHREG, respectively). Model selection procedures were conducted (1) with all significant and notable loci identified from linear regression in multiple linear-regression model selection, (2) with only significant and notable markers identified in both binary and survival analyses using linear regression and survival analysis respectively, and (3) with only significant loci identified in both binary and survival analyses using linear regression and survival analysis respectively. Outcomes from all of the models were compared for congruence among statistically significant markers retained in the final models.

\section{RESULTS}

\section{Clonal hybrid resistance/susceptibility}

The OSU $\times$ ARL $(n=24)$ and OSU $\times$ CW $(n=22)$ hybrid groups exhibited marked differences in resistance to Ceratomyxa shasta (Fig. 1), highly statistically significant for both proportions surviving $\left(\chi^{2}=56.74\right.$, $\mathrm{df}=1, \mathrm{p}<0.0001)$ and for days to death $\left(\chi^{2}=46.55\right.$, $\mathrm{df}=1, \mathrm{p}<0.0001)$. All OSU $\times$ ARL hybrids had died by $53 \mathrm{~d}$ post-exposure. By contrast, only $4 \%(\mathrm{n}=1)$ of the OSU $\times \mathrm{CW}$ individuals had died by the termination of the experiment at $90 \mathrm{~d}$. All of the controls exposed at the same time as clonal hybrids died before termination of the study.

\section{Doubled-haploid susceptibility}

Forty-six percent of the doubled haploids $(\mathrm{n}=50)$ died from Ceratomyxa shasta infection following exposure in the Willamette River. A total of 5 individuals died of other causes, as determined by the absence of C. shasta spores from intestinal scrapings. These indi- 


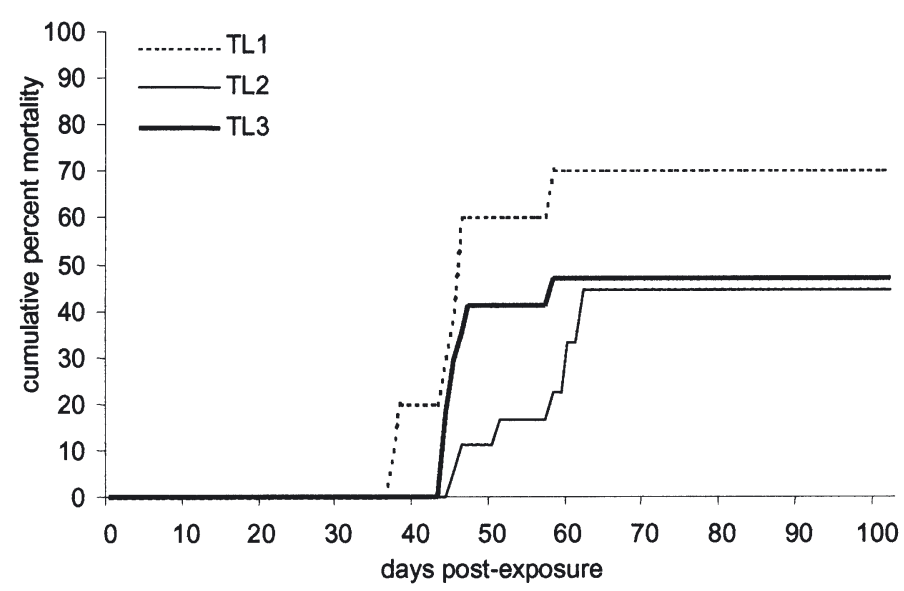

Fig. 2. Oncorhynchus mykiss infected by Ceratomyxa shasta. Cumulative percentage mortality for 3 doubled-haploid families from a cross between the Oregon State University (OSU) and Clearwater River (CW) clonal lines. The 3 families are named according to the egg source (TL: Troutlodge) and number for the outbred female used to produce doubled haploids by androgenesis from sperm of an OSU $\times$ CW hybrid

viduals were removed from all analyses, leaving a sample size of 45 individuals for further analyses. All individuals that survived to the end of the study did not have C. shasta spores evident from posterior intestinal scraping, and no individuals died in the last $40 \mathrm{~d}$ prior to the termination of the experiment; this indicates a resistance to either initial infection or replication of the parasite. Eighty-eight percent $(n=30 / 34)$ of control fish died from C. shasta infection by the termination of the experiment. No statistical difference was observed in either proportions surviving $\left(\chi^{2}=2.16, \mathrm{df}=2, \mathrm{p}=\right.$ 0.34 ) or survival functions $\left(\chi^{2}=0.49, \mathrm{df}=1, \mathrm{p}=0.48\right)$ among doubled-haploid families produced from different outbred females (Fig. 2). Pooling all female families, the proportions of individuals that died and survived were not significantly different $\left(\chi^{2}=0.52, \mathrm{df}=1\right.$, $\mathrm{p}=0.47$ ), indicating that a test of the hypothesis of a single Mendelian locus was warranted.

\section{Genetic linkage map}

With 45 segregants in the analysis, linkage mapping of 343 AFLP markers resulted in 38 linkage groups. The map has a total length of $934.1 \mathrm{cM}$, with 313 total markers mapped, and an average inter-marker distance of $5.7 \mathrm{cM}$ (164 unique positions). Of these linkage groups, 26 had greater than 3 markers, many with clusters characteristic of the large linkage groups identified in another study in our laboratory (OSU $\times$ Arlee map; Young et al. 1998, Nichols et al. 2003). In total, 27 of the OSU $\times \mathrm{CW}(\mathrm{OC})$ linkage groups directly matched to linkage groups in our OSU $\times$ Arlee $(\mathrm{OA})$ rainbow trout map (Young et al. 1998, Nichols et al. 2003). Two additional groups for which significant single-marker associations were identified (OC17 and OC22) were inferred to matches with OA linkage groups based on mapping in another OSU $\times$ CW mapping population (data not shown). Syntenies with linkage groups in the OA map indicate that several of the small groups in this study are not linked to larger groups to which they belong. AFLP marker syntenies between this and the OA maps indicate that the linkage groups containing significant markers (for association with Ceratomyxa shasta) each match to unique OA linkage groups (different chromosomes). However, it is expected that the OSU $\times$ ARL map would have one more linkage group than that of OSU $\times \mathrm{CW}$ based on the karyotypes of these clonal lines (Ristow et al. 1998, C. Ostberg pers. comm.). The separate linkage groups in the OA map that would be adjoined in the OC map are not known at this time.

\section{Marker-trait analyses}

Single-locus hypothesis

Since equal proportions of doubled haploids were resistant and susceptible, the single Mendelian locus hypothesis was tested with linkage mapping as described above. An attempt to assign the binary resistance/susceptibility phenotype to the framework map failed to find significant linkage with any of the linkage groups identified in this study, even at LOD 1.0.

\section{Multiple-locus hypothesis}

Because the binary trait failed to map to any linkage group, marker-trait associations were tested using models where survival was expressed in both binary and days-to-death terms. Linear regression for the binary trait revealed 9 AFLP markers that were significantly $(p \leq 0.05)$, and 21 other markers that were notably ( $p \leq 0.10$ ), associated with resistance. The same result was obtained with logistic regression. When single-marker or comparison-wise significance values from permutation tests were used to determine significance for linear regression, the same results were obtained, except that 11 of the notable markers from linear regression reported above were significant, and 6 additional markers not previously identified above were notable (data not shown). None of the markers in these analyses surpassed the empirical genome-wide threshold value for $\mathrm{p} \leq 0.10$ that was determined by the permutation test. Marker significance hereafter refers to the significance value 


\section{Linkage group $^{1}$}

OC5
(OA-V)

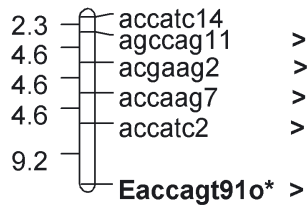

OC17

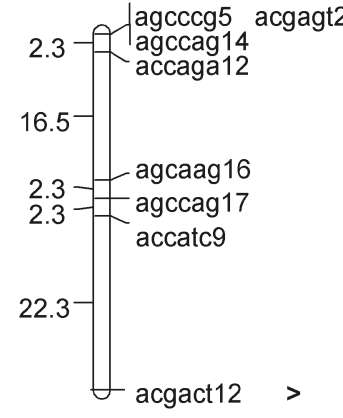

OC19

(OA-XIX)

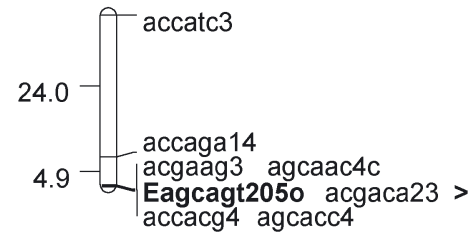

OC21

(OA-XXI)
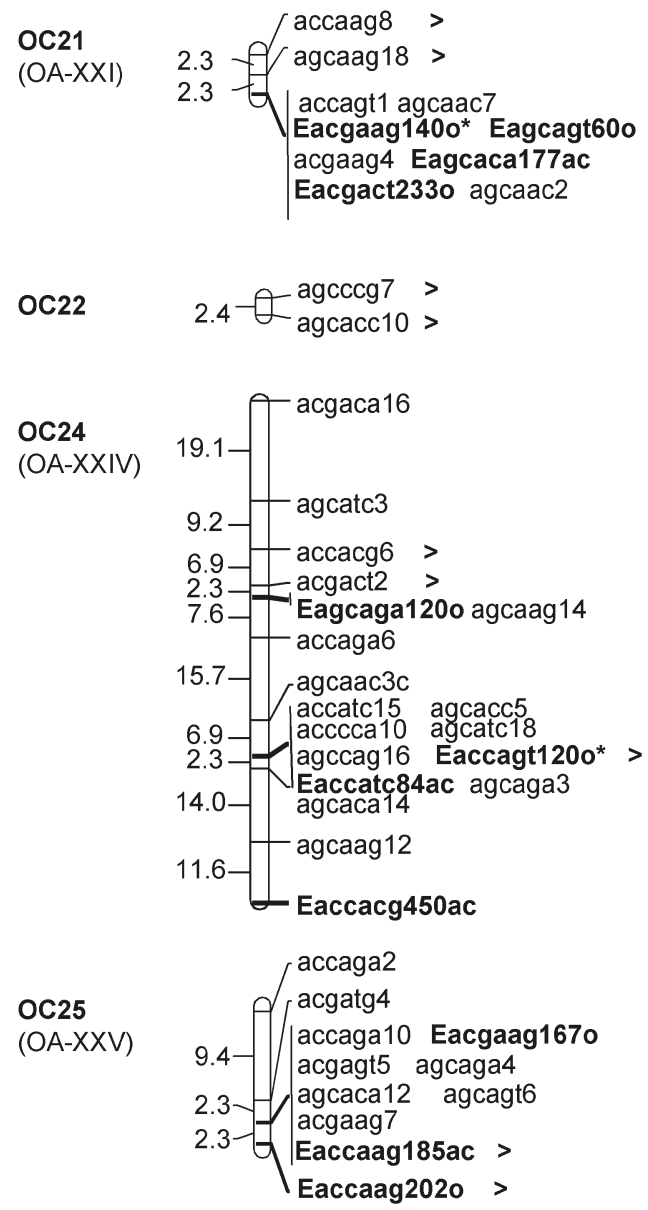

\section{$\underline{\text { Significance }}^{2} \quad$ Correlation $^{3}$}

$\mathrm{n} \mid \mathrm{n}$

${ }^{*} \mid-$

$\mathrm{n} \mid-$

$\mathrm{n} \mid-$

none

** $\mid$ *

OC22

none

OC25

${ }^{*} \mid{ }^{*}$

${ }^{*}{ }^{* *}$

OC17, OC25

one tion.

Significant markers were located on 4 different linkage groups (OC17, OC21, OC22, OC25), and many of the notable markers were linked to these significant markers (Fig. 3). Three additional linkage groups contained markers that were notably associated with resistance (OC5, OC19, and OC24). OC5 and OC19 markers were notable by PROC REG, but significant by permutation tests. Four unlinked markers (agcacc2, acgaga9, agcaga6, acccca11) showed significant and 1 unlinked marker (agcccg6) showed notable single marker associations. None of these unlinked markers have been scored in our reference map cross (Young et al. 1998, Nichols et al. 2003) or have been scored or linked in prior linkage mapping efforts within this cross (Nichols unpubl. data). Survival analysis revealed 6 AFLP markers (Fig. 3) significantly associated with genotypic differences in days-todeath survival curves. These markers were the same as 6 of the 9 significant markers revealed with linear regression. A total of 10 additional markers

Fig. 3. Oncorhynchus mykiss resistance to Ceratomyxa shasta. Linkage groups for which single-marker associations were found in analyses of binary trait and survival (days to death) analyses for C. shasta resistance. ${ }^{1}$ Linkage group gives both the name for the Oregon State University $(\mathrm{OSU}) \times$ Clearwater River (CW) (abbreviated together as OC) group, as well as the reference map linkage group (OSU $\times$ Arlee; OA) to which it is syntenic. Markers in bold are those conferring synteny among the OC and OA maps. ${ }^{2}$ Singlemarker significance results are denoted by symbols in the format: linear regression I survival analysis, where ${ }^{* *} \mathrm{p}<0.01,{ }^{*} 0.01<$ $\mathrm{p}<0.05, \mathrm{n}=0.05<\mathrm{p}<0.10$ (notable) and - indicates $p>0.10$. Three unlinked markers were also significantly associated in these analyses $(p<0.05)$. ${ }^{3}$ Significant intercorrelation of markers among linkage groups are noted as determined by Kendall correlation coefficients 
Table 1. Multiple-regression model selection final results, including proportion of variance in survival explained by all final terms in the model $\left(\mathrm{r}^{2}\right.$ - linear regression only), parameter estimates $\left(\beta_{\mathrm{i}}\right)$, their standard errors (SE), and significance values $(\mathrm{p})$ when: (a) all significant and notable markers were tested in multiple linear regression model selection; (b) when only markers that were significant or notable in both survival and linear regression analyses were included in model selection; and (c) when only markers that were significant in both analyses were included in model selection. Only markers significant in the final models are shown. 'Marker' column superscripts denote the significance of markers in single-marker linear/survival analyses $\left({ }^{*} p<0.05\right.$; $\left.\mathrm{n}=0.05<\mathrm{p}<0.10_{i}-=\mathrm{p}>0.10\right)$

\begin{tabular}{|c|c|c|c|c|c|c|c|c|c|c|c|}
\hline \multirow[t]{3}{*}{$\begin{array}{l}\text { Linkage } \\
\text { group }\end{array}$} & \multirow[t]{3}{*}{ Marker } & \multirow{2}{*}{\multicolumn{2}{|c|}{$\begin{array}{c}\mathrm{a} \\
\text { All notable and } \\
\text { significant markers } \\
\text { from linear regression } \\
\text { Linear }\left(\mathrm{r}^{2}=0.72\right)\end{array}$}} & \multicolumn{4}{|c|}{$\begin{array}{c}\mathrm{b} \\
\text { Markers significant or } \\
\text { notable in both survival } \\
\text { and linear regression analysis }\end{array}$} & \multicolumn{4}{|c|}{$\begin{array}{c}\text { C } \\
\text { Markers significant from } \\
\text { both linear regression } \\
\text { and survival analyses }\end{array}$} \\
\hline & & & & \multicolumn{2}{|c|}{ Linear $\left(r^{2}=0.48\right)$} & \multicolumn{2}{|c|}{ Survival } & \multicolumn{2}{|c|}{ Linear $\left(r^{2}=0.41\right)$} & \multicolumn{2}{|c|}{ Survival } \\
\hline & & $\beta_{\mathrm{i}}( \pm \mathrm{SE})$ & $\mathrm{p}$ & $\beta_{\mathrm{i}}( \pm \mathrm{SE})$ & $\mathrm{p}$ & $\beta_{\mathrm{i}}( \pm \mathrm{SE})$ & $\mathrm{p}$ & $\beta_{\mathrm{i}}( \pm \mathrm{SE})$ & $\mathrm{p}$ & $\beta_{\mathrm{i}}( \pm \mathrm{SE})$ & $\mathrm{p}$ \\
\hline OC5 & $\operatorname{acgaag} 2 \mathrm{n} /-$ & $0.23 \pm 0.09$ & 0.019 & - & - & - & - & - & - & - & \\
\hline OC17 & $\operatorname{acgact} 12 * * *$ & $-0.38 \pm 0.11$ & 0.0016 & $-0.47 \pm 0.14$ & 0.0013 & $1.4 \pm 0.5$ & 0.0067 & $-0.42 \pm 0.15$ & 0.007 & $1.2 \pm 0.5$ & 0.0253 \\
\hline OC21 & $\operatorname{agcaag} 18^{\mathrm{n} /-}$ & $0.28 \pm 0.10$ & 0.0089 & - & - & - & - & - & - & - & - \\
\hline $\mathrm{OC} 24$ & accacg6 n/- & $0.32 \pm 0.09$ & 0.0014 & _- & - & _- & _- & - & _- & _- & _- \\
\hline Unlinked & $\operatorname{acgaga} 9 * / *$ & $0.34 \pm 0.10$ & 0.0014 & $0.34 \pm 0.12$ & 0.0095 & $-1.6 \pm 0.5$ & 0.0015 & $0.38 \pm 0.13$ & 0.005 & $-1.6 \pm 0.5$ & 0.0015 \\
\hline Unlinked & acccca11 */* & $0.46 \pm 0.10$ & $<0.0001$ & $0.37 \pm 0.13$ & 0.0085 & $-1.2 \pm 0.6$ & 0.038 & - & - & - & - \\
\hline Unlinked & $\operatorname{agcacc} 2 * / n$ & $-0.40 \pm 0.10$ & 0.0003 & $-0.37 \pm 0.13$ & 0.0059 & $1.1 \pm 0.5$ & 0.031 & - & - & - & - \\
\hline Unlinked & agcaga6 ${ }^{* / *}$ & - & - & - & - & - & - & $-0.32 \pm 0.13$ & 0.018 & $1.1 \pm 0.5$ & 0.0398 \\
\hline
\end{tabular}

were notably associated with days to death, encompassing a subset of the same notable and significant markers identified with linear regression; 3 of the significant days-to-death survival markers were unlinked, and the other 3 were located on linkage groups OC17 and OC22 (Fig. 3). Of the significant or notable markers that were unlinked at LOD 3.0, 2 (agcccg6 and accca11) localized to the ends of 2 other distinct linkage groups (OC1 and OC20) when the LOD score for linkage was decreased to 2.0. Three linkage groups, OC5, OC21, and OC24, possess markers that were significantly and notably associated with the binary trait, but none of these markers were associated with days to death (Fig. 3). Interval mapping by linear regression failed to find significant loci. Kendall correlation coefficients among significant and notable loci reveal that markers on different linkage groups are significantly correlated (Fig. 3). Significant inter-correlations among loci on different linkage groups may indicate non-independent segregation among loci on some groups due to small sample sizes.

Multiple-marker model selection in both linear regression and survival analyses revealed that variation in resistance is associated with multiple loci (Table 1). In selecting a model for variation in the binary resistance phenotype using all significant and notable markers from linear regression, 7 markers significantly contributed to variation in the binary resistance phenotype $(F=12.22$, df $=7, \mathrm{p}<0.0001)$ when 19 markers were included for model selection (Table 1). Three unlinked markers (acgaga9, acccca11, and agcacc2) and 1 marker from each of OC5, OC17, OC21, and OC24 explained $66 \%$ of the variation in the binary sur- vival phenotype. Of the unlinked loci, 2 (agcacc2 and acccca11) are notably correlated $(0.05<\mathrm{p}<0.10)$, and it is possible, with the addition of more markers and/or more individuals, that these markers may be linked. However, these loci have opposite effects in the final models, and even if linked, it appears that these loci contribute uniquely to the variation in resistance to Ceratomyxa shasta. In models tested using only notable and/or significant loci that were identified by both binary and survival single-marker analyses (using 11 markers), multiple-marker linear and survival analyses identified the same significant loci (Table 1). The terms in this linear regression model explained $48 \%$ of the variation in binary survival $(F=8.40, \mathrm{df}=4$, $\mathrm{p}<0.0001)$. Four loci were found to significantly contribute to variation in resistance -1 on OC17 and 3 unlinked markers. When testing markers significant in both single-marker survival and linear regression models (6 markers), 3 loci were identified for significant contribution to variation in resistance - the same locus on OC17 and 2 unlinked markers. The linear regression model, considering only significant markers from both types of analyses, explained $41 \%$ of the variation in binary survival $(F=8.99$, df $=3, \mathrm{p}<0.0001)$. Only 1 locus was discordant among all analyses. The unlinked marker, agcaga6, significantly contributed to variation in resistance when only significant markers were considered, but was not significant when notable markers were considered in all other models. This marker was significantly correlated with markers on OC21, OC22, and OC25, but was not correlated with any of the other markers that dropped out of the model when only significant markers were considered. Al- 
though these models have removed the redundancy of multicollinear markers, it is not clear why agcaga6 was retained in the model, as it was not correlated with any other locus that had a negative effect on survival.

In all cases where markers were significant in the models tested, linear regression and survival analyses agree on the relative magnitude and effect of each locus. Two loci have an observed negative effect on survival. Those with a minus sign for linear regression coefficients suggest that the CW genotype can decrease survival; these same loci have a positive regression coefficient in the survival analysis, which will increase the value of the hazard function and thus decrease survival for individuals with the CW genotype. In the analysis where all significant and notable loci are included in linear-regression model selection, a greater number of loci show positive effects, whereby individuals with the CW genotype at those loci have greater survivability. When markers that were not congruent between linear and survival analyses, or that were not significant in both analyses, were not included in model selection, fewer loci with a positive effect on survival with the CW genotype were found in model selection.

\section{DISCUSSION}

We have revealed multiple genomic loci associated with resistance to Ceratomyxa shasta in Oncorhynchus mykiss following a short-term in situ exposure to the pathogen. Evidence that the binary trait failed to map to a single location, despite segregation that indicated a simple Mendelian model, together with multiple single-marker associations on different linkage groups, confirms polygenic control of resistance in this species. The identification of multiple loci in this study is consistent with results from inheritance studies by Ibarra et al. $(1992,1994)$, who concluded that the trait was controlled by more than 1 gene. This study has not only confirmed that inheritance is polygenic, but has identified potential linkage groups and markers associated with $C$. shasta resistance in these $O$. mykiss clonal lines.

Resistance, expressed as absolute survival (binary trait) and as days to death (survival analysis), for the most part revealed the same significant and notable loci for associations with Ceratomyxa shasta resistance. The only difference observed between binary trait and survival analyses was the identification of significant and notable loci found on OC5, OC21, and OC24 with linear regression. The detection of these significant loci in the binary trait analysis, but not in the survival analysis, suggests that different mechanisms may be involved in absolute resistance or mor- tality versus the time course of $C$. shasta pathogenesis. Ibarra et al. (1994) have proposed 2 modes of resistance to C. shasta: resistance may occur by control of the initial stages of infection or may relate to the time course of pathogenesis once infected. However, with such a small sample size in the study herein, the occurrence of false positives may be greater in the binary trait analysis since lesser information was contained in the binary coding of the phenotype than with the daysto-death coding. Without larger samples sizes and more fine-scaled mapping, we cannot determine whether the detection of notable binary trait loci that were not detected in survival analysis is an artifact of small samples sizes, or whether there are real differences in the modes of resistance associated with each locus.

We have used simple statistical models, extended by previous investigators for the analysis of binary trait (McIntyre et al. 2001) and survival data (Symons et al. 2002), to assess the number of loci involved in Ceratoтуха shasta resistance. Although significant single markers would not have been significant when corrected for multiple tests, these markers guided our model selection procedures from which the final models clearly show that multiple loci significantly contribute to a substantial portion of the variation in C. shasta resistance. We could not estimate the distance of significant markers to putative trait loci because statistical programs to do so for survival and binary trait data are either not available for doubledhaploid designs (Xu \& Atchley 1996, Symons et al. 2002), or are not specified for simultaneous tests with multiple loci (McIntyre et al. 2001). Interval mapping by linear regression failed to detect significant binary trait loci, a result likely due to the small sample size in this study and associated failure to exceed the conservative threshold designed to decrease the rate of detecting false positives with multiple-interval tests. Previous investigators (Vermerris \& McIntyre 1999, Symons et al. 2002) have used survival analysis to improve inference made on marker-trait associations where trait distributions are skewed, but we failed to detect novel loci with this type of analysis. In fact, more significant loci were revealed with binary trait analysis - a result, as previously stated, that could be attributed either to differences in the genes involved in absolute vs. time-course of resistance, or to the power of this study to detect significant loci with any type of analysis. Multiple regression model selection revealed highly significant markers on several linkage groups in all models tested, but due to multicollinearity of these markers with markers on other linkage groups, we cannot ascertain which of these loci are truly linked to genes involved in C. shasta resistance. We have noted co-segregation (by correlation) of loci on distinct link- 
age groups, an inability to link smaller groups to larger ones, and an inability to link significant single markers to any linkage group. These phenomena are most likely a result of the inability to break co-segregation of groups by chance with a limited number of segregations, and the low probability that rare recombination events will be observed. More detailed studies with greater numbers of individuals will aid in more precise localization of loci associated with $C$. shasta resistance in these Oncorhynchus mykiss clonal lines.

Based upon syntenies with our reference rainbow trout map (Young et al. 1998, Nichols et al. 2003), we have found that significant markers associated withCeratomyxa shasta on OC21 in this cross are located on the same linkage group as a novel immune-type receptor gene (NITR2, Yoder et al. 2002) and an immunoreceptor tyrosine-based inhibition motif-bearing C-type lectin (TCL-2; Zhang et al. 2001). Both of these genes are located in or very near the cluster of AFLP markers on OC21, but the association of these potential candidate genes in C. shasta resistance cannot be ascertained without genotyping and mapping in this cross. Ozaki et al. (2001) have revealed 2 genomic loci associated with infectious pancreatic necrosis virus (IPNV) in rainbow trout. One locus associated with IPNV resistance maps to the same linkage group (OC22) as C. shasta resistance loci in this cross, but the overlap of genes involved in resistance to the 2 very different pathogens cannot be determined without more fine-mapping of loci in both studies. Linkage group OC22 in this cross corresponds to linkage group OA-XXII, which is syntenic to linkage group $\mathrm{C}$ in the outcross mapping panel used in their study, as determined by our updated reference map (Nichols et al. 2003) that consolidates the 2 major published rainbow trout linkage maps (Young et al. 1998, Sakamoto et al. 2000).

This is the first study to evaluate the genetic basis of resistance to any Myxozoan parasite using molecular markers. We have found multiple genome regions involved in resistance to Ceratomyxa shasta in these Oncorhynchus mykiss clonal lines. Multiple regression models suggest that at least 3 loci contributed to the variation in C. shasta resistance, but the precise location of these loci cannot be determined in our present study. This study suggests that some markers may be developed for testing of associations with C. shasta resistance in completed outbred inheritance studies (Bartholomew et al. 2001), for which molecular markers were not yet available. Similar studies using a greater number of individuals and fine mapping of these regions by introgression of resistance alleles into our susceptible clonal line may provide more detailed information on the genes and mechanisms conferring C. shasta resistance and susceptibility.
Acknowledgements. The authors thank L. M. McIntyre for advice on genetic analysis of binary trait data and M. A. Evans for discussions on statistical analyses. Two anonymous reviewers were helpful with comments on an earlier draft of this manuscript. Troutlodge generously provided the eggs for production of clonal lines and doubled haploids. Thanks to P. A. Wheeler for production and shipping of fish for disease challenges. M. H. Whipple and H. A. Lorz assisted with disease challenges at Oregon State University. This research was supported by a grant to G.H.T. from the National Science Foundation (IBN-0082773).

\section{LITERATURE CITED}

Bartholomew JL (1998) Host resistance to infection by the Myxosporean parasite Ceratomyxa shasta: a review. J Aquat Anim Health 10:112-120

Bartholomew JL, Whipple MJ, Stevens DG, Fryer JL (1997) The life cycle of Ceratomyxa shasta, a myxosporean parasite of salmonids, requires a freshwater polychaete as an alternate host. J Parasitol 83:859-868

Bartholomew JL, Whipple MJ, Campton D (2001) Inheritance of resistance to Ceratomyxa shasta in progeny from crosses between high- and low-susceptibility strains of rainbow trout (Oncorhynchus mykiss). Bull Nat Res Inst Aquacult Suppl 5:71-75

Basten CJ, Weir BS, Zeng Z-B (2002) QTL Cartographer, Version 1.16. Department of Statistics, North Carolina State University, Raleigh

Broman KW, Wu H, Sen S, Churchill GA (2003) R/qtl: QTL mapping in experimental crosses. Bioinformatics 19: 889-890

Buchanan DV, Sanders JE, Zinn JL, Fryer JL (1983) Relative susceptibility of four strains of summer steelhead to infection by Ceratomyxa shasta. Trans Am Fish Soc 112: 541-543

Haley CS, Knott SA (1992) A simple regression method for mapping quantitative trait loci in line crosses using flanking markers. Heredity 69:315-324

Hemmingsen AR, Holt RA, Ewing RD, McIntyre JD (1986) Susceptibility of progeny from crosses among three stocks of coho salmon to infection by Ceratomyxa shasta. Trans Am Fish Soc 115:492-495

Ibarra AM, Hedrick RP, Gall GAE (1992) Inheritance of susceptibility to Ceratomyxa shasta (Myxozoa) in rainbow trout and the effect of the length of exposure on the liability to develop ceratomyxosis. Aquaculture 104:217-229

Ibarra AM, Hedrick RP, Gall GAE (1994) Genetic analysis of rainbow trout susceptibility to the myxosporean, Ceratomуха shasta. Aquaculture 120:239-262

Lander ES, Botstein B (1989) Mapping Mendelian factors underlying quantitative traits using RFLP linkage maps. Genetics 121:185-199

Lander ES, Green P, Abrahamson J, Barlow A, Daly MJ (1987) MAPMAKER: an interactive computer package for constructing primary genetic linkage maps of experimental and natural populations. Genomics 1:174-181

McIntyre LM, Coffman CJ, Doerge RW (2001) Detection and localization of a single binary trait locus in experimental populations. Genet Res 78:79-92

Nichols KM, Young WP, Danzmann RG, Robison BD, and 15 others (2003) A consolidated genetic linkage map for rainbow trout (Oncorhynchus mykiss). Anim Genet 34: 102-115

Ozaki A, Sakamoto T, Khoo S, Nakamura K, Coimbra MRM, Akutsu T, Okamoto N (2001) Quantitative trait loci (QTLs) 
associated with resistance/susceptibility to infectious pancreatic necrosis virus (IPNV) in rainbow trout (Oncorhynchus mykiss). Mol Genet Genomics 265:23-31

Parsons JE, Thorgaard GH (1984) Induced androgenesis in rainbow trout. J Exp Zool 231:407-412

Rebai A (1997) Comparison of methods for regression interval mapping in QTL analysis with non-normal traits. Genet Res 69:69-74

Ristow SS, Grabowski LD, Ostberg C, Robison B, Thorgaard GH (1998) Development of long-term cell lines from homozygous clones of rainbow trout. J Aquat Anim Health 10:75-82

Robison BD, Wheeler PA, Sundin K, Sikka P, Thorgaard GH (2001) Composite interval mapping reveals a major locus influencing embryonic development rate in rainbow trout (Oncorhynchus mykiss). J Hered 92:16-22

Sakamoto T, Danzmann RG, Gharbi K, Howard P and 8 others (2000) A microsatellite linkage map of rainbow trout (Oncorhynchus mykiss) characterized by large sexspecific differences in recombination rates. Genetics 155: 1331-1345

Symons RCA, Daly MJ, Fridlyand J, Speed TP, Cook WD, Gerondakis S, Harris AW, Foote SJ (2002) Multiple genetic loci modify susceptibility to plasmocytoma-related morbidity in Eu-v-abl transgenic mice. Proc Nat Acad Sci 99:11299-11304

Vermerris W, McIntyre LM (1999) Time to flowering in brown

Editorial responsibility: Carey Cunningham, Aberdeen, UK midrib mutants of maize: an alternative approach to the analysis of developmental traits. Heredity 83:171-178

Visscher PM, Haley CS, Knott SA (1996) Mapping QTLs for binary traits in backcross and $F_{2}$ populations. Genet Res 68:55-63

Voorrips RE (2002) MapChart: software for the graphical presentation of linkage maps and QTL. J Hered 93:77-78

Whipple MJ, Gannam AL, Bartholomew JL (2002) Lack of a prophylactic effect of orally administered glucan and fumagillin on naturally acquired infection with Ceratomyxa shasta in juvenile rainbow and steelhead trout (Oncorhynchus mykiss). N Am J Aquacult 64:1-9

Xu S, Atchley WR (1996) Mapping quantitative trait loci for complex binary diseases using line crosses. Genetics 143: $1417-1424$

Yoder JA, Mueller MG, Nichols KM, Ristow SS, Thorgaard GH, Ota T, Litman GW (2002) Cloning novel immune-type inhibitory receptors from the rainbow trout, Oncorhynchus mykiss. Immunogenetics 54:662-670

Young WP, Wheeler PA, Coryell VH, Keim P, Thorgaard GH (1998) A detailed linkage map of rainbow trout produced using doubled haploids. Genetics 148:839-850

Zhang H, Nichols K, Thorgaard GH, Ristow SS (2001) Identification, mapping, and genomic structural analysis of an immunoreceptor tyrosine-based inhibition motif-bearing C-type lectin from homozygous clones of rainbow trout (Oncorhynchus mykiss). Immunogenetics 53:751-759

Submitted: January 10, 2003; Accepted: June 9, 2003 Proofs received from author(s): August 18, 2003 